Article

\title{
Combined Optimal Torque Feedforward and Modal Current Feedback Control for Low Inductance PM Motors
}

\author{
Roland Kasper * and Dmytro Golovakha \\ Chair of Mechatronics, Otto von Guericke University, 39106 Magdeburg, Germany; dmytro.golovakha@ovgu.de \\ * Correspondence: roland.kasper@ovgu.de
}

Received: 7 October 2020; Accepted: 22 November 2020; Published: 25 November 2020

check for updates

\begin{abstract}
Small sized electric motors providing high specific torque and power are required for many mobile applications. Air gap windings technology allows to create innovative lightweight and high-power electric motors that show low phase inductances. Low inductance leads to a small motor time constant, which enables fast current and torque control, but requires a high switching frequency and short sampling time to keep current ripples and losses in an acceptable range. This paper proposes an optimal torque feedforward control method, minimizing either torque ripples or motor losses, combined with a very robust and computation-efficient modal current feedback control. Compared to well-known control methods based on the Clarke-Park Transformations, the proposed strategy reduces torque ripples and motor losses significantly and offers a very fast implementation on standard microcontrollers with high robustness, e.g., against measurement errors of rotor angle. To verify the accuracy of the proposed control method, an experimental setup was used including a wheel hub motor built with a slotless air gap winding of low inductance, a standard microcontroller and GaN (Gallium Nitride) Power Devices allowing for high PWM switching frequencies. The proposed control method was validated first by correlation of simulation and experimental results and second by comparison to conventional field-oriented control.
\end{abstract}

Keywords: optimal control; modal current control; feedforward torque control; feedback current control; torque ripples and loss minimization; low inductance permanent magnet motor

\section{Introduction}

Manufacturers of electric vehicles or electric planes require electric motors with small size, lightweight construction and maximum efficiency, providing at the same time high torque and power. For low-power applications $(<200 \mathrm{~W})$, electrical motor weight can be reduced by air gap winding designs offered, e.g., by Faulhaber and Maxxon [1]. A new slotless air gap winding design for a 15-inch wheel-hub motor providing high power of $40 \mathrm{~kW}$ at a speed of $1500 \mathrm{rpm}$ and a very low weight of $20 \mathrm{~kg}$ is presented in References [2-4]. Wheel hub motors take a new step in technology of electric vehicles as they allow to create completely new designs of mobile applications without a lot of the mechanics used today and a lot more room for passengers and baggage. High specific power and torque densities are the main indicators of the quality of wheel hub motors. This way, the very lightweight wheel hub motors based on air gap windings [2-4] are most promising.

Usually, motors are based on slotted windings, which significantly increase the motor weight and reduce specific torque and power density. Slotless air gap winding design reduces the weight of electric motors, as it avoids a big part of stator back-iron needed for conventional slotted machines. Additionally, a very high torque can be generated without any torque ripple induced by slotted geometry. However, low number of turns and low iron volume in air gap winding designs lead 
to very low phase inductances of only several $\mu \mathrm{H}$, which makes control a challenge $[4,5]$. For this reason, a high pulse width modulation (PWM) switching frequency of about $100 \mathrm{kHz}$ is needed to keep current ripples, and accordingly torque ripples and associated losses, in a demanded range. In addition, the motor control must be adapted to low phase inductance and high PWM frequency for microcontrollers with a very small sampling step size.

Depending on the phase current waveform, conventional control methods for permanent magnet (PM) motors can be divided into two groups: based on the block commutation [5-9] and based on the Clarke-Park Transformations [10-16]. The first one is usually used for low-cost and low-power applications because of low manufacturing costs and simple design. On the other hand, this leads to a high torque ripple waveform caused by non-ideal commutation currents [5-9]. This disadvantage is even more significant for PM motors with low inductance due to the inverse relationship between phase inductances and current ripples. Trigonometric Clarke-Park Transformations transfer phase currents into a torque generating Q-part and a field generating D-part (DQ control), dependent on rotor position [10-16]. This gives the opportunity for smoother phase commutations, which decreases current and torque ripple but assumes pure sinusoidal waveforms for B-field (magnetic flux density) and phase currents. For high sampling frequencies, calculation of transformations is a high burden for the microcontroller. If the B-field consists of significantly higher harmonics, they should be compensated by adding additionally higher harmonic parts to the phase currents. This method may be unacceptable for high-frequency control, and only iterative approaches are known [10-14].

This article proposes a combined optimal torque feedforward and modal current feedback control method for PM motors with very low phase inductance. Previously, in References $[17,18]$, a high-frequency Combined Optimal Torque and Modal Current Control (OTMIC) was proposed for low inductance PM motors focused on the minimization of motor losses. In this article, this method for loss minimization is described as mathematically complete. Further, it is extended to torque ripple minimization to give a powerful optimization method flexibly adaptable to different goals. Limitations of References $[17,18]$ given by growing size of symbolic expressions will be avoided by a new matrix formulation of the algorithm that allows for adaptive real-time applications. It is possible to switch online between both optimization strategies to fulfill changing demands depending on certain operation points (e.g., low speed $\rightarrow$ minimal torque ripple, high speed $\rightarrow$ minimal loss). In case of motors based on air gap winding designs, motor B-field is nearly independent of speed, torque and phase currents. This conclusion was used to explicitly take into consideration all harmonics of B-field for torque control. In an off-line optimization step, optimal current values for a set of electric angles are stored in a table for on-line usage under hard real-time conditions. By means of a very robust modal current control approach, which works independently on rotor angle, the 3-phase current control problem can be reduced to a 2-phase modal one, based on a linear model of the PM machine with constant coefficients. It becomes evident that the non-controllable state (one of the 3-phase currents) can be eliminated without any pre-conditions, e.g., on B-field waveform, and all statements on stability are global using this approach. Additionally, modal transformation matrices are constant, especially independent of electrical angle and any physical parameter. This reduces computation effort significantly compared, e.g., to Park-Clarke Transformation, where transformation matrices change with the angle. Errors of angle measurement or estimation have only a very limited effect on current control. Thus, independence of motor parameters increases the robustness of the transformation due to any physical changes, further allowing for a very fast software implementation on a low-cost microcontroller with an acceptable sampling step size. Optimization as well as control problem can be solved symbolically, allowing for very fast and adaptive implementations.

In the second section of this article, a new approach of PM motor control based on the mathematical model of slotless air gap winding PM motor with very low phase inductance is presented. The proposed method combines modal feedback control to guarantee the desired stability, dynamics and robustness margins with optimal feedforward control to minimize important motor features like ohmic losses or torque ripple. The last section shows the experimental and simulation results of the proposed method 
and comparison of the proposed control with conventional Field-Oriented Control (FOC) based on DQ Clarke-Park Transformations (DQ control). A PM wheel hub motor with slotless air gap windings and very small inductances (near $1.5 \mu \mathrm{H}$ per phase) was used as a test object.

\section{Design of the Proposed Combined Optimal Feedforward Torque and Modal Current Feedback Control for Low Inductance PM Motors}

\subsection{Operation Principle of PM Motor with Very Low Phase Inductance}

The dynamic model of a PM motor with slotless air gap winding is obtained considering the following assumptions [3]:

1. Rotor consists of surface-mounted permanent magnets.

2. No cogging torque due to slotless design.

3. Harmonic magnetic field density generated by permanent magnets.

4. B-field harmonics and inductance are constant, e.g., independent from speed and phase currents due to very small current-induced magnetic fields of the windings.

5. Stator has symmetric 3-phase star-connected windings commutated by a 3-phase inverter (B6-bridge).

A model is shown in Figure 1, where $R$-phase resistance, $L, M$-phase self and mutual inductance, $\underline{e}=\left[\begin{array}{lll}e_{a} & e_{b} & e_{c}\end{array}\right]^{T}$-phase back-EMFs (back electromotive force), $\underline{u}=\left[\begin{array}{lll}u_{a} & u_{b} & u_{c}\end{array}\right]^{T}$, $\underline{i}=\left[\begin{array}{lll}i_{a} & i_{b} & i_{c}\end{array}\right]^{T}$-phase voltages and currents, $u_{S}$-voltage at star point and $u_{D C}, i_{D C}$-input direct voltage, current to PM motor inverter [2-4].

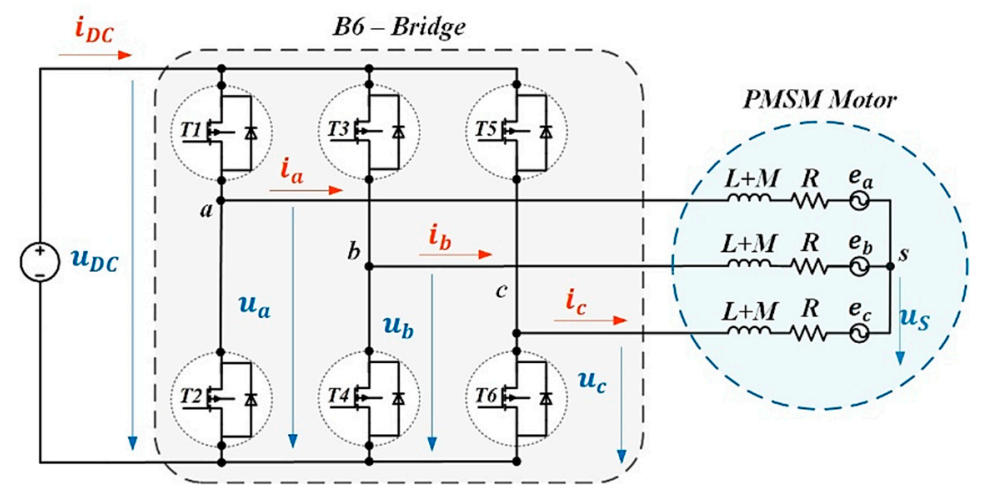

Figure 1. B6-bridge-fed 3-phase permanent magnet (PM) drive connections.

Considering the Y-connection, Kirchhoff's first Law

$$
i_{a}+i_{b}+i_{c}=0
$$

and Kirchhoff's second Law

$$
\left[\begin{array}{l}
u_{a} \\
u_{b} \\
u_{c}
\end{array}\right]=\left[\begin{array}{lll}
R & 0 & 0 \\
0 & \mathrm{R} & 0 \\
0 & 0 & \mathrm{R}
\end{array}\right]\left[\begin{array}{c}
i_{a} \\
i_{b} \\
i_{c}
\end{array}\right]+\left[\begin{array}{ccc}
\mathrm{L} & -M & -M \\
-M & \mathrm{~L} & -M \\
-M & -M & \mathrm{~L}
\end{array}\right]\left[\begin{array}{c}
d i_{a} / d t \\
d i_{b} / d t \\
d i_{c} / d t
\end{array}\right]+\left[\begin{array}{c}
e_{a} \\
e_{b} \\
e_{c}
\end{array}\right]+\left[\begin{array}{l}
1 \\
1 \\
1
\end{array}\right] u_{S}
$$

or more compact

$$
\underline{u}=\underline{R} \underline{i}+\underline{L} d \underline{d i} / d t+\underline{e}+\underline{u}_{S}
$$

state the mathematical model of a 3-phase PM motor. The 3-phase back-EMF values

$$
\underline{e}=\omega k_{M} \underline{B}(\varphi),
$$


depend on $\omega$-the mechanical angular velocity of the rotor, $k_{M}$ - a motor geometric constant and $\underline{B}=\left[\begin{array}{lll}B_{a}(\varphi) & B_{b}(\varphi) & B_{c}(\varphi)\end{array}\right]^{T}$-the vector of averaged B-fields acting on air gap winding phases [3], which itself depends on $\varphi$-the electrical angle. Obviously, $B_{a}(\varphi)=B(\varphi), B_{b}(\varphi)=B\left(\varphi-\frac{2 \pi}{3}\right)$ and $B_{c}(\varphi)=B\left(\varphi-\frac{4 \pi}{3}\right)$ are given by the harmonic magnetic field density generated by permanent magnets, calculated by:

$$
B(\varphi)=\sum_{k} b_{k} \sin (k \varphi)
$$

as a function of electric angle and odd harmonic coefficients, $b_{k}$. Either FE analysis of the underlying magnetic circuit or line-fitting of measured back-EMF values give the B-field harmonic coefficients with very good accuracy [2-4].

Electrical torque is generated by Lorentz force [2-4]:

$$
T_{E}=k_{M}\left[\begin{array}{lll}
B_{a} & B_{b} & B_{c}
\end{array}\right]\left[\begin{array}{c}
i_{a} \\
i_{b} \\
i_{c}
\end{array}\right]=k_{M} \underline{B}^{T} \underline{i}
$$

acting on the airgap winding. Loss torque:

$$
T_{L}=d \omega+c
$$

collects linear mechanical friction together with back-iron and airgap winding eddy losses into the linear friction coefficient $d$ and mechanical coulomb friction together with back-iron hysteresis losses into the constant friction coefficient $c$. It will be noted that loss torque $T_{L}$ and resulting loss power depends only on speed and is independent of motor current. Electrical torque $T_{E}$ reduced by loss torque $T_{L}$ gives motor output torque:

$$
T_{M}=T_{E}-T_{L} .
$$

Equations (1)-(8) describe the motor model in terms of back-EMF, output torque and phase currents based on information of angular position $\varphi$, angular velocity $\omega$, B-field harmonics and motor parameters $k_{M}, d$ and $c$. Torque Equation (6) shows that the adaptation of phase current waveforms taking into account the B-field harmonics is the key to motor torque control [2-4].

Phase currents $i(\varphi)$, like B-field, can also be defined as harmonic functions of electrical angle $\varphi$ :

$$
i(\varphi)=\sum_{k} a_{k} \sin (k \varphi)
$$

Cosine terms are omitted. If included, they would vanish during optimization. From (1), we get additional constraints:

$$
a_{3 k}=0
$$

for current coefficients. Average electrical torque:

$$
T_{A}=\frac{1}{2 \pi} \int_{0}^{2 \pi} T_{E} d \varphi=\frac{3}{2} k_{M} \sum_{k} a_{k} b_{k}
$$

can then be expressed by Fourier coefficients of phase current and B-Field. Using Equations (5) and (8)-(11), in this paper will be proposed a new method to generate optimal phase currents to generate a given torque $T_{\text {Ref }}$ for an arbitrary harmonic B-field, while minimizing either the motor losses or alternatively while minimizing the output torque ripples. 


\subsection{Optimal Control for Minimal Motor Losses}

Generation of a given torque $T_{\text {Ref }}$ with maximum efficiency or, equivalently expressed, with minimum losses $P_{L}$ is a typical design requirement of an electrical motor. Averaged total motor losses consist of ohmic and non-ohmic losses [3]:

$$
\begin{gathered}
P_{L}=\frac{1}{2 \pi} \int_{0}^{2 \pi}\left(R\left(i_{a}^{2}+i_{b}^{2}+i_{c}^{2}\right)+d \omega^{2}+c \omega\right) d \varphi \\
P_{L}=\frac{3 R}{2} \sum_{k} a_{k}^{2}+d \cdot \bar{\omega}^{2}+c \cdot \bar{\omega}
\end{gathered}
$$

where non-ohmic losses depend only on the average angular speed in a pole pair segment:

$$
\bar{\omega}=\int_{0}^{2 \pi} \omega d \varphi \text { and } \bar{\omega}^{2}=\int_{0}^{2 \pi} \omega^{2} d \varphi
$$

and ohmic losses can be expressed by Fourier coefficients of phase currents according to (9). Given demands lead to the mathematical optimization problem for minimization of motor losses $P_{L}$ while generating a demanded torque $T_{R e f}$ :

$$
\text { Min }=P_{L} \quad \text { with constraint } \quad T_{A}=T_{R e f}
$$

for unknown parameters $a_{k}$ of the phase current harmonics based on the given harmonics $b_{k}$ of the B-field. The solution of this quadratic optimization problem can be determined analytically, e.g., by combining cost function and constraints in a Lagrange function, calculating derivatives and solving first-order conditions for unknown coefficients:

$$
a_{m}=\frac{2}{3} \frac{T_{R e f}}{k_{M}} \frac{b_{m}}{\bar{b}^{2}}
$$

with the sum of squares of selected harmonics of B-field:

$$
\bar{b}^{2}=\sum_{m} b_{m}^{2}
$$

and the selection indices:

$$
m=1,5,7,11,13,17,19,23 \ldots
$$

Remaining parameters $a_{k}$ are zero. Selection indices show that some odd harmonics of phase current $\{3,9,15, \ldots\}$ will not contribute to torque. The optimal current waveforms can be calculated from (15) for each electrical angle taking into account the harmonics of B-field, motor constant $k_{M}$ and given reference torque $T_{\text {Ref }}$. This approach achieves that each demanded torque can be generated with minimum motor losses for arbitrary B-field and regardless of motor speed. Air gap winding PM motor design allows to have nearly ideally constant B-field harmonics with minor sensitivity to temperature changes. If necessary, e.g., for application to other motor types, the effect of temperature on B-field and phase resistances can be easily incorporated into the model and adapted online.

\subsection{Optimal Control for Minimal Torque Ripples}

Generation of a given torque $T_{\text {Ref }}$ with minimum torque ripples is another typical design requirement of an electrical motor, especially if vibrations and noise are critical. Torque ripple is defined as mean square deviation between electrical torque $T_{E}$ and average torque $T_{A}$ :

$$
T_{R}^{2}=\frac{1}{2 \pi} \int_{0}^{2 \pi}\left(T_{E}-T_{A}\right)^{2} d \varphi
$$


In this case, optimal current can be determined solving the optimization problem:

$$
\text { Min }=T_{R}^{2} \quad \text { with constraint } \quad T_{A}=T_{R e f}
$$

Inserting current definition (9) into the integral (18), the optimization problem stated in (19) again can be solved analytically by formulating Lagrange function, calculating derivatives and solving the first-order conditions. Besides this straightforward path, there exists another approach, which leads to more simple expressions and allows further insight into the solution. Starting from (5), (6) harmonics

$$
t_{k}=\frac{1}{\pi} \int_{0}^{2 \pi} T_{E} \cos (6 k \varphi) d \varphi
$$

of electrical torque $T_{E}$ can be determined and collected in vector

$$
\underline{t}=\underline{A} \underline{a}
$$

with matrix

$$
\underline{A}=\frac{2}{3} k_{M}\left[\begin{array}{cccccccc}
2 b_{1} & 2 b_{5} & 2 b_{7} & 2 b_{11} & 2 b_{13} & 2 b_{17} & 2 b_{19} & 2 b_{23} \\
b_{7}-b_{5} & b_{11}-b_{1} & b_{13}+b_{1} & b_{17}+b_{5} & b_{19}+b_{7} & b_{23}+b_{11} & b_{13} & b_{17} \\
b_{13}-b_{11} & b_{17}-b_{7} & b_{19}-b_{5} & b_{23}-b_{1} & b_{1} & b_{5} & b_{7} & b_{11} \\
b_{19}-b_{17} & b_{23}-b_{13} & -b_{11} & -b_{7} & -b_{5} & -b_{1} & b_{1} & b_{5} \\
-b_{23} & -b_{19} & -b_{17} & -b_{13} & -b_{11} & -b_{7} & -b_{5} & -b_{1} \\
0 & 0 & -b_{23} & -b_{19} & -b_{17} & -b_{13} & -b_{11} & -b_{7} \\
0 & 0 & 0 & 0 & -b_{23} & -b_{19} & -b_{17} & -b_{13} \\
0 & 0 & 0 & 0 & 0 & 0 & -b_{23} & -b_{19}
\end{array}\right]
$$

and vector of current harmonics

$$
\underline{a}=\left[a_{1}, a_{5}, a_{7}, a_{11}, a_{13}, a_{17}, a_{19}, a_{23}\right]
$$

for an example with 23 harmonics. Only average value at zero and multiples of the sixth harmonic appear. All other coefficients are zero. Matrix $\underline{A}$ is square and has full rank. Defining the torque harmonics

$$
\underline{t}=\left[2 T_{R e f}, 0,0,0,0,0,0,0\right]
$$

in a way that the zero coefficient is given by the demanded torque $T_{R e f}$ and all other torque harmonics vanish, delivers the solution for the above-mentioned optimization problem by solving the linear system of equations defined by (21). For practical applications, a much smaller number of harmonics than given in (23) will be sufficient. The larger number presented here helps to demonstrate the structure of the matrix. It is important to state that also the torque ripples minimization leads to an analytical solution for optimal currents that is well suited for online and adaptive applications.

\subsection{Modal Current Control}

So far, we have two feedforward strategies to implement either loss minimal or torque ripples minimal operation of the motor. Both strategies rely on current control, which is able to deal with higher current harmonics, which means also with higher frequencies. A modal current control method, exploiting all structural benefits of given control problem and allowing for very high-speed control, will be presented in more detail in this section. The complete model of the PM motor with air gap winding is defined by (1)-(3). Node Equation (1) shows that only 2 of the 3 phase currents are independent. Taking this dependency into account, (3) can be written as

$$
\underline{G}(\underline{u}-\underline{e})=\underline{R} \underline{i}+\underline{L} d \underline{i} / d t
$$


with

$$
\underline{G}=\frac{1}{3}\left[\begin{array}{ccc}
2 & -1 & -1 \\
-1 & 2 & -1 \\
-1 & -1 & 2
\end{array}\right]
$$

For simplicity, back-EMF $\underline{e}$ can be neglected and later added to input voltage $\underline{u}$ as a feedforward compensation or simply treated as a disturbance for the controller. This coupled system for the 3 phase currents $\underline{i}$ can be completely decoupled with a modal transformation

$$
\left[\begin{array}{c}
J_{\Sigma} \\
J_{1} \\
J_{2}
\end{array}\right]=\underbrace{\frac{1}{3}\left[\begin{array}{ccc}
1 & 1 & 1 \\
-1 & -1 & 2 \\
-1 & 2 & -1
\end{array}\right]}_{\underline{W}}\left[\begin{array}{l}
i_{a} \\
i_{b} \\
i_{c}
\end{array}\right]
$$

applied to system (25) in a way that modal inductance matrix

$$
\underline{\Lambda}=\underline{W} \underline{L}^{-1}=\left[\begin{array}{ccc}
L-2 M & 0 & 0 \\
0 & L+M & 0 \\
0 & 0 & L+M
\end{array}\right]
$$

gets diagonal. Phase currents $\underline{i}$ are transformed to modal currents $J=\left[\begin{array}{lll}J_{\Sigma} & J_{1} & J_{2}\end{array}\right]^{T}$. Considering (1) and the first row of the transformation matrix defined in (27), modal current $J_{\Sigma}$ will be zero. Thus, only 2 out of 3 modal currents associated with the modal inductances $L+M$ are relevant. The decoupled modal system

$$
\frac{1}{3}\left[\begin{array}{ccc}
0 & 0 & 0 \\
-1 & -1 & 2 \\
-1 & 2 & -1
\end{array}\right]\left[\begin{array}{l}
u_{a} \\
u_{b} \\
u_{c}
\end{array}\right]=\left[\begin{array}{lll}
R & 0 & 0 \\
0 & R & 0 \\
0 & 0 & R
\end{array}\right]\left[\begin{array}{l}
J_{\Sigma} \\
J_{1} \\
J_{2}
\end{array}\right]+\left[\begin{array}{ccc}
L-2 M & 0 & 0 \\
0 & L+M & 0 \\
0 & 0 & L+M
\end{array}\right]\left[\begin{array}{l}
d J_{\Sigma} / d t \\
d J_{1} / d t \\
d J_{2} / d t
\end{array}\right]
$$

shows that modal current $J_{\Sigma}$ is not controllable as the associated row of the modal input matrix is completely zero. The remaining 2 modal currents, $J_{1}$ and $J_{2}$, come from 2 identical first-order systems with time constant $\frac{L+M}{R}$. Eliminating non-controllable current $J_{\Sigma}$ gives the reduced controllable system

$$
\underbrace{\frac{1}{3}\left[\begin{array}{ccc}
-1 & -1 & 2 \\
-1 & 2 & -1
\end{array}\right]}_{\underline{\Gamma}}\left[\begin{array}{l}
u_{a} \\
u_{b} \\
u_{c}
\end{array}\right]=\left[\begin{array}{ll}
R & 0 \\
0 & R
\end{array}\right]\left[\begin{array}{l}
J_{1} \\
J_{2}
\end{array}\right]+\left[\begin{array}{cc}
L+M & 0 \\
0 & L+M
\end{array}\right]\left[\begin{array}{l}
d J_{1} / d t \\
d J_{2} / d t
\end{array}\right]
$$

Thus, the 3-phase current control problem is reduced to the control of two completely identical but decoupled first-order systems, associated with modal currents $J_{1}$ and $J_{2}$. The redundant total current $J_{\Sigma}$ displays any errors in current control loop and can be used as an indicator of the quality of the system online. Modal currents $J_{1}$ and $J_{2}$ are not connected and can be controlled completely independent by modal input voltages

$$
\underline{V}=\left[\begin{array}{l}
V_{1} \\
V_{2}
\end{array}\right]=\underline{\Gamma} \cdot \underline{u},
$$

as shown in Figure 2. This simple separation based on the modal transformation given in (27) does not use any trigonometric calculations based on the rotor angle as in conventional Clarke-Park Transformations, is independent of motor parameters and is well defined for any shape of B-field. 


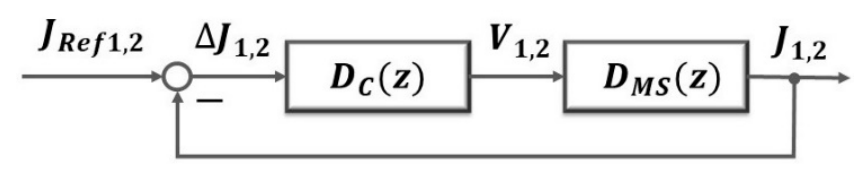

Figure 2. Modal control loop for modal currents $J_{1,2}$.

According to (30). motor transfer function is given by

$$
G_{M}(s)=\frac{1 / R}{1+\frac{L+M}{R} S} .
$$

For high-speed control of low inductance PM motors with high number of poles, current sensor dynamics must be included in the control loop with a first-order sensor model

$$
G_{S}(s)=\frac{1}{1+T_{S} S}
$$

and sensor time constant $T_{S}$. As a microcontroller-driven B6-bridge generates PWM pulses at frequency $f_{P W M}=1 / \Delta t$ to control motor input voltage and ADC (Analog-to-Digital Converter) samples current sensor signals with the same rate, time continuous transfer function

$$
G_{M S}(s)=G_{M}(s) G_{S}(s)
$$

has to be discretized with that frequency to get the discrete transfer function

$$
D_{M S}(z)=\frac{(\beta-1+(1-\alpha) \delta) z+((\alpha-1) \delta-\alpha) \beta+\alpha}{R(\delta-1)(z-\alpha)(z-\beta)}
$$

with sampling time $\Delta t$, discrete eigenvalues of motor $\alpha=e^{-\frac{\mathrm{R} \Delta t}{\mathrm{~L}+\mathrm{M}}}$, sensor $\beta=e^{-\frac{\Delta t}{T_{S}}}$ and motor/sensor speed ratio $\delta=\frac{\mathrm{L}+\mathrm{M}}{\mathrm{R} T_{S}}$. Very small motor time constant due to low phase inductance allows to achieve very fast current and electrical torque response with requested time constant $T_{\text {Req }}$, which expands the possibilities of design for more complex control systems like dynamic stability control or autonomous driving. To get a controlled system of first order

$$
D(z)=\frac{1-z_{R}}{z-z_{R}}
$$

with requested eigenvalues $z_{R}=e^{-\frac{\Delta t}{T_{R e q}}}$, it is necessary to use a discrete PID (Proportional-Integral-Derivative) controller with filtered derivative action:

$$
D_{C}(z)=K_{P}+\frac{K_{I}}{z-1}+\frac{K_{D}}{N_{D}+\frac{1}{z-1}} .
$$

Control parameters can be determined by comparison of coefficients of discrete closed loop

$$
D(z)=\frac{D_{C}(z) D_{M S}(z)}{1+D_{C}(z) D_{M S}(z)}
$$


and reference transfer function given in (36), as

$$
\begin{aligned}
& K_{P}=\frac{(1-\alpha+\delta(\beta-1)) R\left(z_{R}-1\right)}{(\delta-1)(\beta-1)(\alpha-1)} ; \\
& K_{I}=R\left(z_{R}-1\right) ; \\
& K_{D}=\frac{R \delta\left(z_{R}-1\right)(\beta-\alpha)^{2}}{(\delta-1)^{2}(\beta-1)^{2}(\alpha-1)^{2}} ; \\
& N_{D}=\frac{\beta-1+\delta(1-\alpha)}{(\delta-1)(\beta-1)(\alpha-1)} .
\end{aligned}
$$

A saturation of output and I-part of the PID controller guarantees safe operation in the case of limited supply voltages. Outputs from PID controllers $D_{C}(z)$ are two modal control voltages, $V_{1}$ and $V_{2}$. According to (31), $V_{1}$ and $V_{2}$ must be transformed back to three control voltages $\underline{u}$ by means of a Moore Penrose pseudo-inverse transformation matrix $\underline{\Gamma}^{+}$

$$
\left[\begin{array}{l}
u_{a} \\
u_{b} \\
u_{c}
\end{array}\right]=\underbrace{\left[\begin{array}{cc}
-1 & -1 \\
0 & 1 \\
1 & 0
\end{array}\right]}_{\underline{\Gamma}^{+}}\left[\begin{array}{l}
V_{1} \\
V_{2}
\end{array}\right] .
$$

Modal current control ensures system stability regardless of fluctuations in system parameters caused by changes of motor temperature and other factors. Moreover, it can be implemented for PM synchronous motors with any waveform of B-field. However, it should be noted, that the motor model should satisfy the mathematical model described with Equations (1)-(3), which can be very good when performed for PM motors with air gap windings and also other PM motors.

\subsection{Control Diagram of OTMIC}

The proposed control diagram of Combined Optimal Torque and Modal Current Control (OTMIC) is shown in Figure 3. The control algorithm uses a torque reference $T_{R e f}$ and measured electrical angle $\varphi$ to estimate optimal phase current waveforms for motor loss or torque ripples minimization, $\underline{i}_{\text {Ref }}=\left[\begin{array}{lll}i_{a R e f}(\varphi) & i_{b R e f}(\varphi) & i_{c R e f}(\varphi)\end{array}\right]^{T}$, based on one of the optimization methods described in Sections 3.1 and 3.2. The optimal currents $\underline{i}_{R e f}$ are used as a reference for measured phase currents $\underline{i}$. Current errors $\Delta \underline{i}$ as differences between reference and measured currents are fed to the Modal Current Control described in Section 2.4 and used to generate the control voltages $\underline{u}$, which set PM motor in motion with the optimal torque waveform via a SVPWM (Space Vector Pulse Width Modulation) and a B6-bridge. Architecture of OTMIC control allows to simplify the microcontroller torque/current control loop by using constant matrices with simple coefficients for modal transformation.

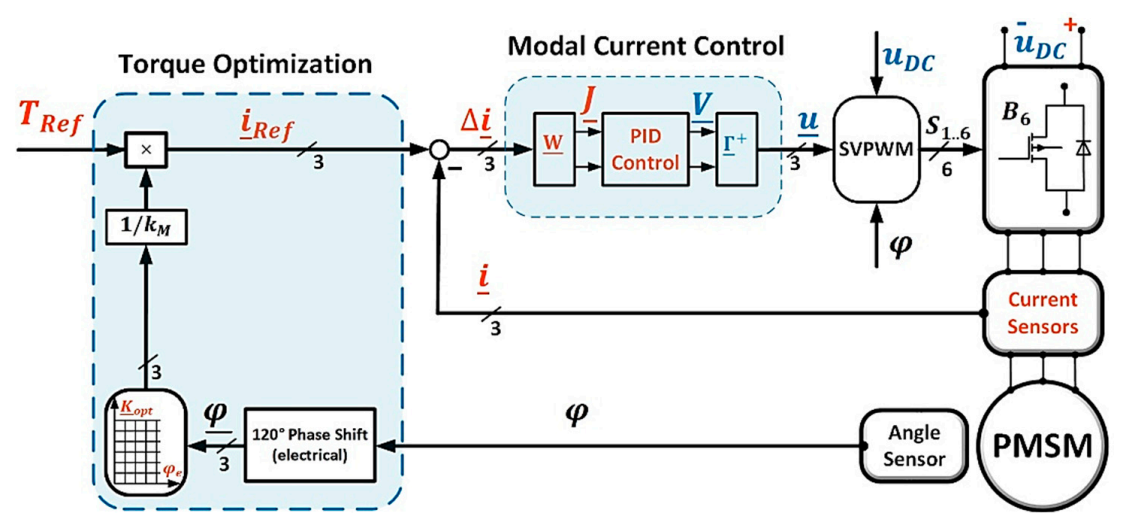

Figure 3. Proposed Combined Optimal Torque and Modal Current Control (OTMIC) diagram of PM motor drive system. 
In addition, optimal reference currents according to (9) and (15)-(17) for minimal Loss Control or from (9) and (21)-(24) for minimal Torque Ripple Control can be calculated off-line for a set of angles and stored in a table. These simplifications allow to implement the control loop with very small step size of $\Delta t<10 \mu$ s on a common low-cost microcontroller.

\section{Units Simulation and Experimental Results}

\subsection{Motivation and Experimental Setup}

A wheel hub motor for a hub-less scooter, shown in Figure 4 on the experimental setup (left above) and in the dissembled state (right) for better demonstration of the air gab winding, will be used for verification of OTMIC control method. The motor has a very low total weight of $2.7 \mathrm{~kg}$, a high nominal power of $4.8 \mathrm{~kW}$ and a nominal torque of $80 \mathrm{Nm}$. More motor data is given in Table 1, where B odd Harmonics are 1st, 3rd, 5th and 7th harmonic numbers. The experimental platform shown in Figure 4 was used to verify the proposed OTMIC method with either minimized motor losses or minimized torque ripples for low inductance PM motors.
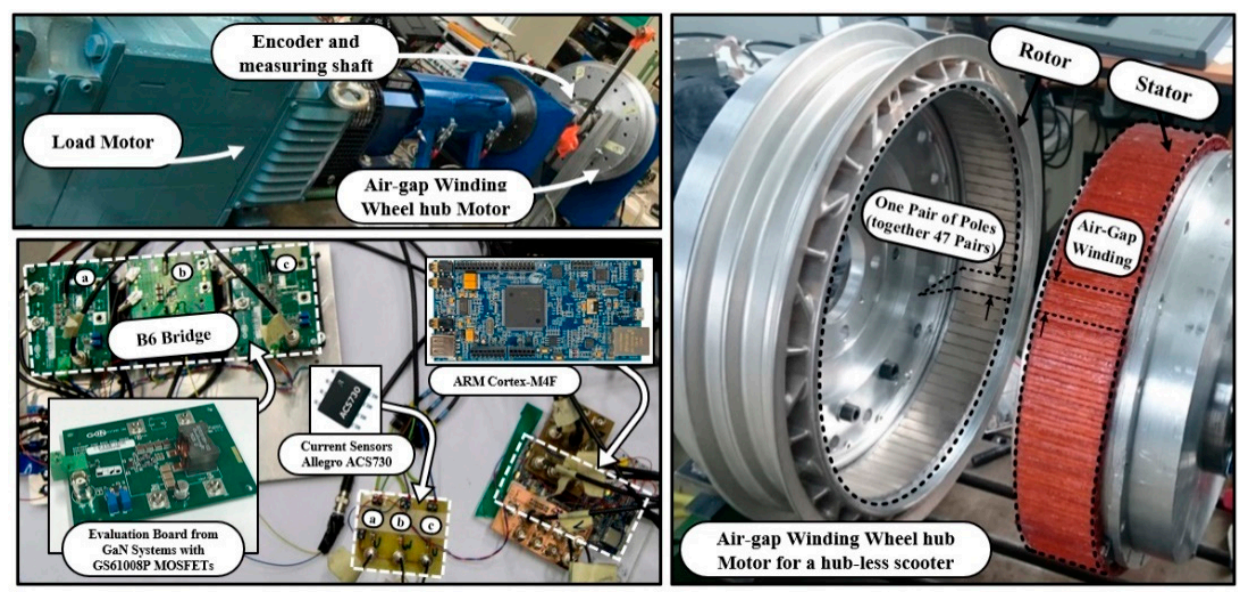

Figure 4. Experimental setup for OTMIC verification [18].

Table 1. Air gab winding wheel-hub motor parameters $[17,18]$.

\begin{tabular}{|c|c|c|c|}
\hline Symbol & Description & Value & UOM \\
\hline \multicolumn{4}{|c|}{ Motor Parameters } \\
\hline$u_{D C}$ & DC voltage & 48 & $V$ \\
\hline$L_{s}+M_{s}$ & phase inductance & 1.5 & $\mu H$ \\
\hline$R_{S}$ & phase resistance & 0.026 & $\Omega$ \\
\hline$t_{M}$ & motor time constant & 58 & $\mu s$ \\
\hline$p$ & number of poles & 94 & - \\
\hline$k_{M}$ & motor constant & 0.304 & $\mathrm{Nm} /(\mathrm{TA})$ \\
\hline$\underline{b}_{k}$ & $\begin{array}{l}\text { B odd harmonics } \\
\text { constant of coulomb }\end{array}$ & \multicolumn{2}{|c|}{$\left[\begin{array}{lllll}1.15 & 0.2 & 0.06 & 0.01\end{array}\right]$} \\
\hline$c$ & $\begin{array}{l}\text { friction and hysteresis } \\
\text { loss }\end{array}$ & 0.0832 & $\mathrm{Nm}$ \\
\hline$d$ & $\begin{array}{l}\text { constant of mechanical } \\
\text { friction and eddy loss }\end{array}$ & 0.0008 & $\mathrm{Nm} \cdot \mathrm{s} / \mathrm{rad}$ \\
\hline \multicolumn{4}{|c|}{ Control Parameters } \\
\hline$K_{P}$ & Proportional term & 0.166 & $V / A$ \\
\hline$K_{I}$ & Integral term & 0.026 & $V / A$ \\
\hline$K_{D}$ & Derivative term & 0.013 & $V / A$ \\
\hline$N_{D}$ & PID filter term & 0.9854 & - \\
\hline$T_{S}$ & sensor time constant & 1 & $\mu s$ \\
\hline$\Delta t$ & sampling time & 10 & $\mu s$ \\
\hline$f_{P W M}$ & switching frequency & 100 & $k H z$ \\
\hline$T_{\text {Req }}$ & requested time constant & 20 & $\mu s$ \\
\hline
\end{tabular}


Due to elimination of stator iron, motor inductance is very low (near $1.5 \mu \mathrm{H}$ per phase). Thus, motor needs an adapted control with high switching frequency of about $100 \mathrm{kHz}$ to keep current ripples and total losses in an acceptable range. Control parameters calculated according to (39) are also given in Table 1.

To drive the motor, six GS61008P MOSFETs (metal-oxide-silicon transistors) from company GaN Systems, able to switch up to $90 \mathrm{~A}$ at a rate up to $300 \mathrm{kHz}$, were connected as a B6-bridge. Due to limited MOSFETs cooling of experimental boards, phase current was restricted to $20 \mathrm{~A}$ for the experiments. Hall-effect-based sensors from Allegro ACS730 were used for phase current measurement with a very small sensor time constant of $1 \mu \mathrm{s}$. Rotor angle is delivered by an encoder using $5 \mathrm{k}$ increments. $100 \mathrm{kHz}$ PWM switching frequency to power the B6-bridge requires a very small step size in the microcontroller (lower than $10 \mu \mathrm{s}$ ). To achieve this requirement, an ARM Cortex-M4F has been used running at $200 \mathrm{MHz}$. It allows to implement the control loop shown in Figure 3 in $8.3 \mu \mathrm{s}$. In comparison to the proposed method, a conventional DQ control needs a minimum of $9.4 \mu \mathrm{s}$, which underlines the effectiveness of the proposed method. This comparison can be seen in Figure 5.

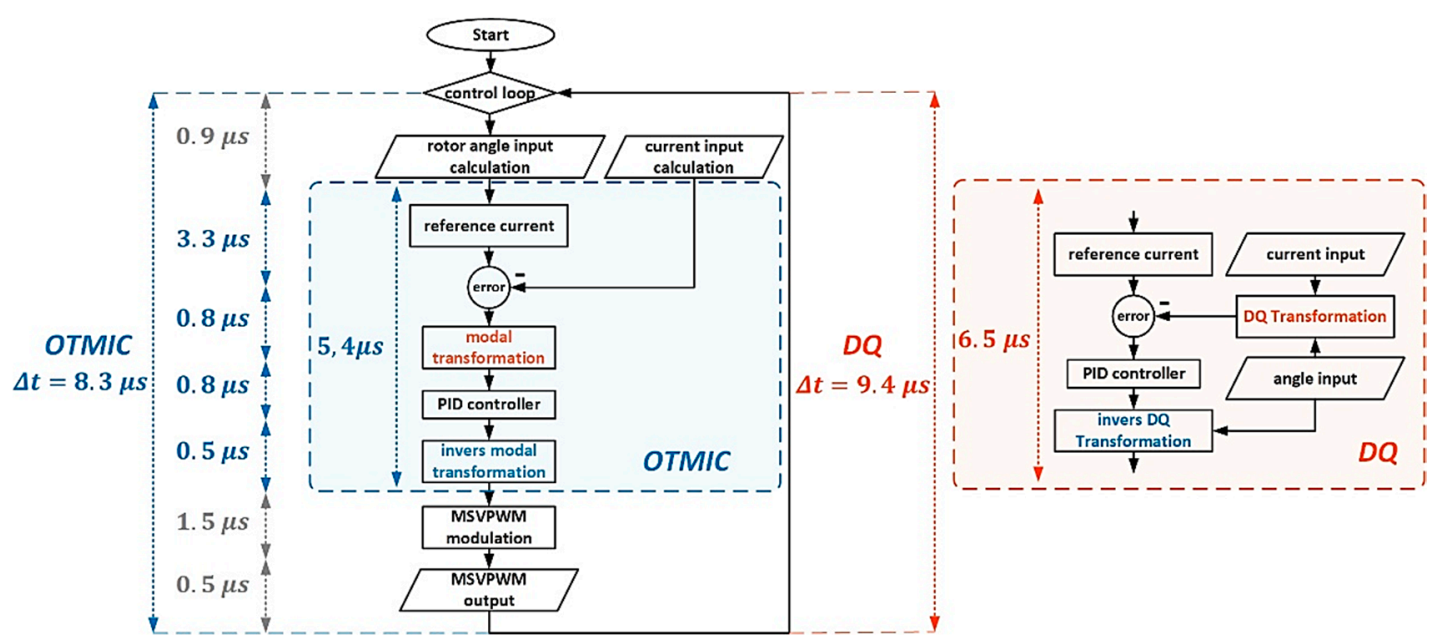

Figure 5. Step response of simulated and measured torque for OTMIC and FOC (DQ).

This means that OTMIC control can be calculated faster and in addition, gives the optimal torque in contrast to FOC (DQ) based on the Clarke-Parke Transformations.

\subsection{Verification of the Proposed Method}

The simulation results presented in this study have been performed through Matlab/Simulink environment based on the mathematical model of the PM motor described in Section 2 and the control model presented in Section 3. Conventional DQ field-oriented vector control used as a reference to the proposed OTMIC was also implemented in the experiment to control the wheel hub motor, with the parameters in Table 1.

The motor dynamic behavior can be verified by means of step response of electrical torque $T_{E}$. As shown in Figure 6, measured and simulated torque of proposed OTMIC and conventional DQ methods were carried out according to zero velocity, mechanically fixed rotor and $5 \mathrm{Nm}$ reference torque $T_{R e f}$ (green). Because torque sensor bandwidth was too low for this experiment, electrical torque generated in air gap winding (grey) was calculated using (6) based on the values of measured currents and the electric angle with a given motor geometric constant $k_{M}$, and compared with the simulation for OTMIC (blue) and DQ (red) control methods. As can be seen form Figure 6, the difference between both methods is minimal due to the very low motor time constant. The exciting fast torque step response within $35 \mu$ s underlines the very high dynamics of this type of motor. The slight difference between simulation and measurement is due to parasitic inductances and capacitances in power lines 
and supply. This can be easily considered in the motor model but was not done in this experiment to show the robustness of the control.

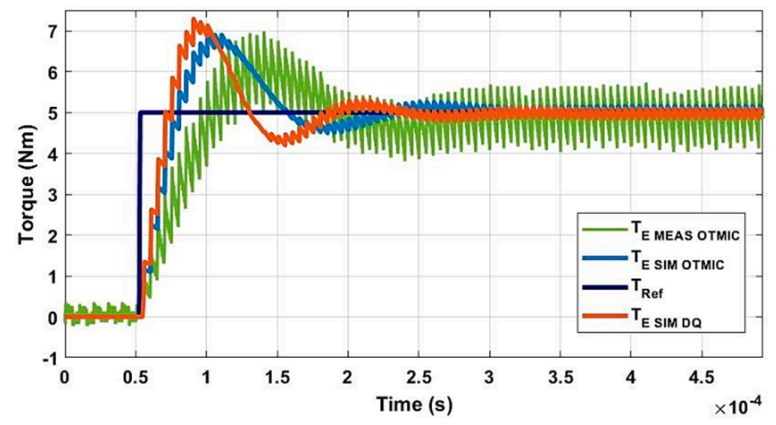

Figure 6. Step response of simulated and measured torque for OTMIC and DQ.

For a more detailed demonstration of the basic principles of the proposed control algorithm, Figure 7 shows the results of modeling the ideal optimal current waveforms to minimize the torque ripples (OTMIC TRO) and losses (OTMIC LO) for two waveforms of B-Field (Case A with odd harmonic coefficients $b_{k}$ from Table 1 and Case B with $b_{k}$ from Reference [19], which result from a change in the air gap between stator and rotor). For a more detailed analysis, the torque RMS (root mean square) and efficiency value are also presented for a sinusoidal current of conventional DQ control based on the Clarke-Park Transformations. Figure 7 shows the simulation results of an ideal system without any errors in the phase current or rotor position measurements. As can be seen, the optimal phase current waveforms are able to the minimize torque ripples or maximize motor efficiency based on the Equations (6) and (12). The advantages of the OTMIC TRO are seen very well in Figure 7. In an ideal system, OTMIC TRO eliminants any torque fluctuations for any waveform of the B-field. OTMIC with loss optimization (LO) changes the phase current waveform and improves loss rates and efficiency of the system with increased torque ripples. The advantages of the OTMIC LO in comparison with the standard DQ method are visible only in the presence of significant parasitic harmonics in the form of a B-field. Simply said, the ideal sinusoidal B-field leads to the same torque ripples and motor loss for every method.
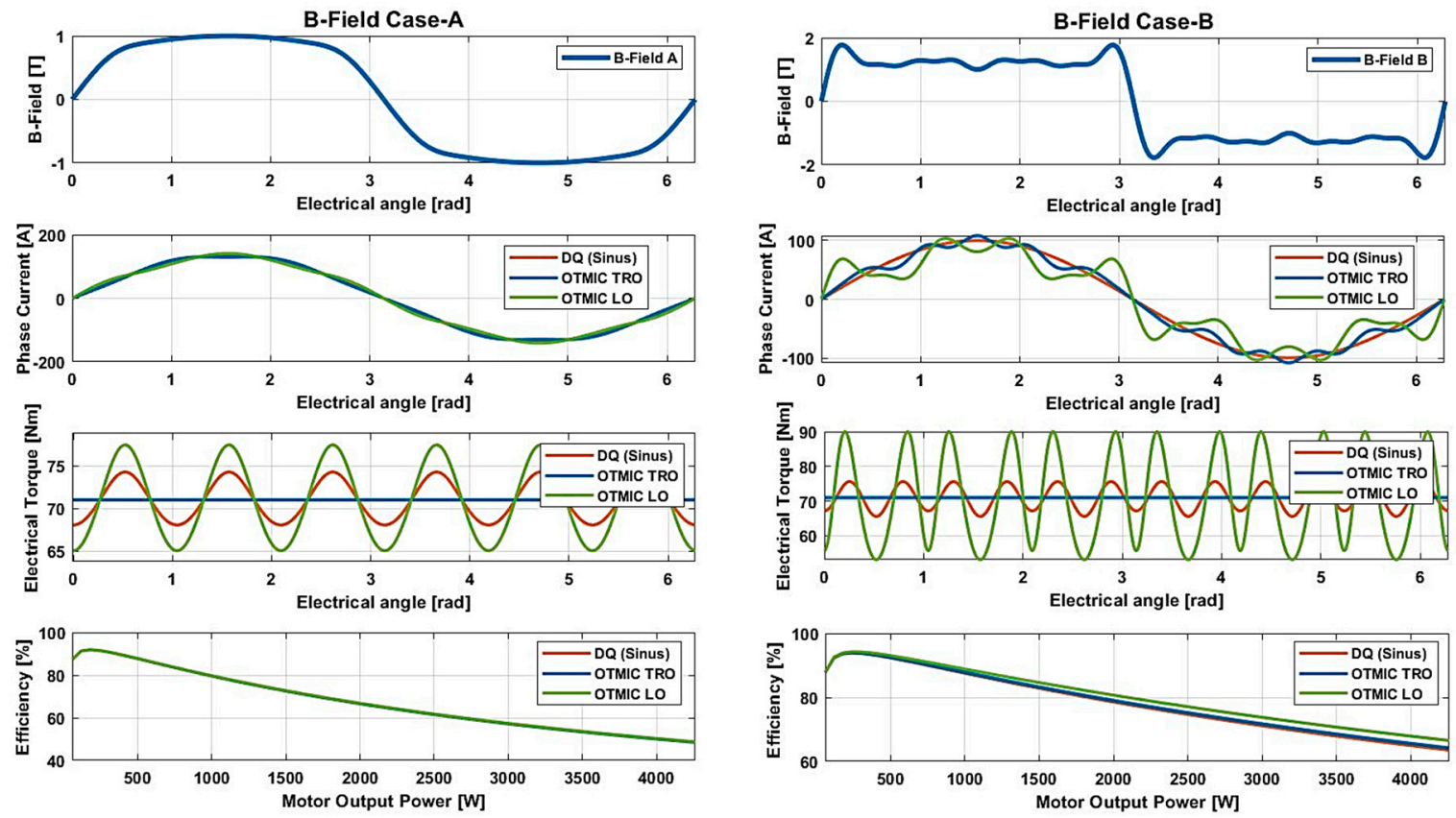

Figure 7. Working principles of the proposed OTMIC control algorithm for two B-Field waveforms. 
Simulated and measured phase currents and torque results during normal operation of proposed OTMIC control with minimization of torque ripple (TRO Figure 8a-simulation, Figure $9 a-$ measurement) and losses (LO Figures $8 b$ and $9 b$ ) as well as a conventional vector DQ control (DQ Figures $8 \mathrm{c}$ and $9 \mathrm{c}$ ) correspond to the references: $\omega=8 \mathrm{rad} / \mathrm{s}$ and $\mathrm{T}_{\text {Ref }}=10 \mathrm{Nm}$. The behavior of phase currents and torque for both OTMIC optimizations given in Figure 9a,b has a slight asymmetry caused by speed fluctuations of load machine and errors in angle measurements, which could not be completely avoided in experimental setup. The current and torque harmonic distribution for the aforementioned control approaches are shown in Figure $8 \mathrm{~d}$ for simulation and in Figure $9 \mathrm{~d}$ for measured results. The amplitude of each harmonic component is normalized relative to that of a fundamental one. The FFT (Fast Fourier transform) analyses of simulation currents and torques underline the main idea of the proposed method. As shown in Figure 8d (left), the phase current waveforms of OTMIC have 5th and 7th harmonics expected to conventional control. On the other hand, the torque waveform of OTMIC with torque ripple optimization TRO (blue) is deprived of such harmonics, which leads to the very small torque fluctuations as opposed to DQ control, as shown in Figure 8d (right).

(a)
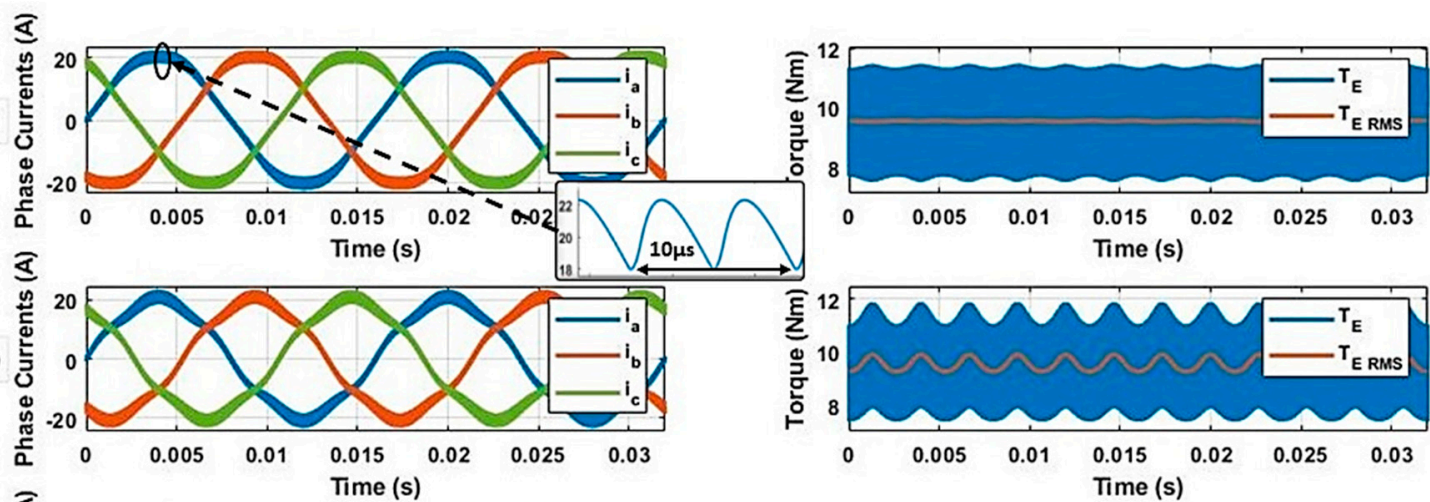

(c)
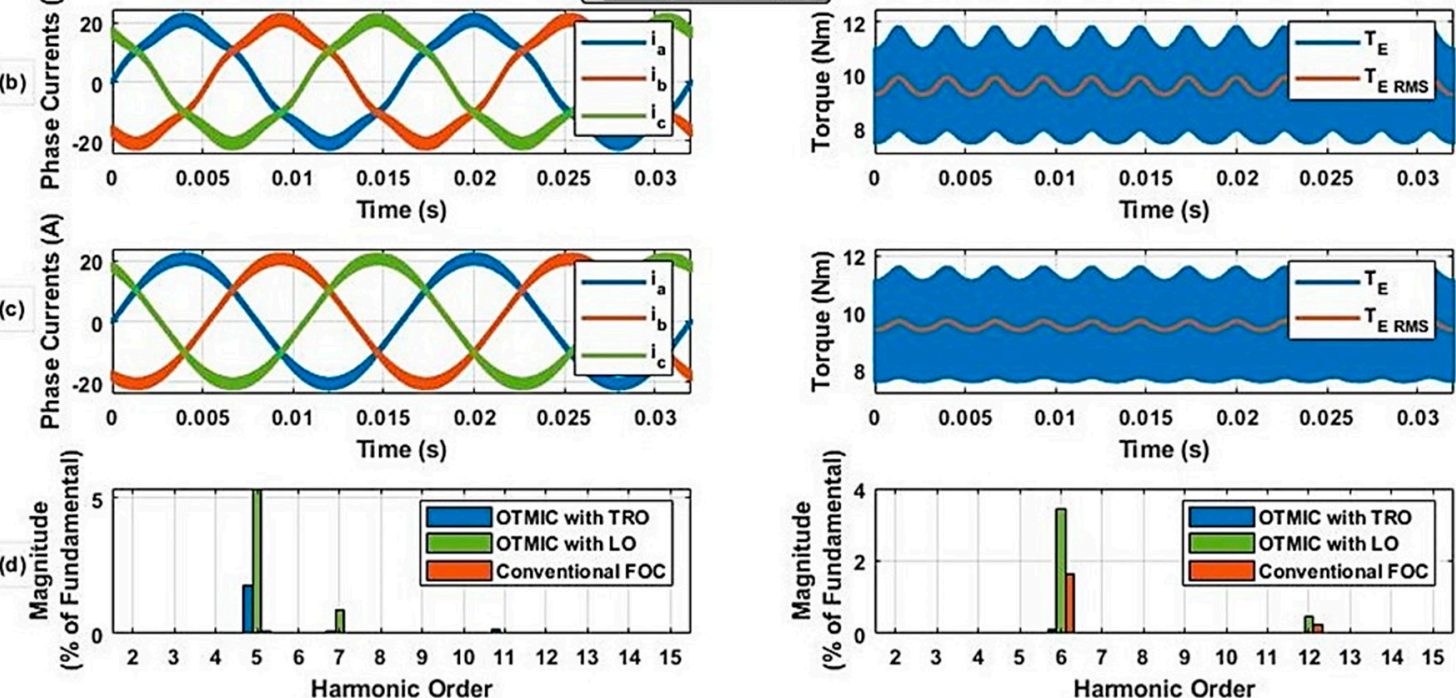

Figure 8. Simulation results of stator current and torque of: (a) OTMIC with torque ripples optimization, (b) OTMIC with losses optimization, (c) conventional DQ control and (d) harmonic spectrum of phase current (left) and torque (right) for every method [18].

The harmonic spectrum of measured phase current with OTMIC control shown in Figure $9 \mathrm{~d}$ (left) has parasitical 2nd, 4th and 6th harmonics due to inaccuracies in experimental setup, mainly caused by interactions of control system and load machine, but they are less than $1 \%$ of that of the fundamental one. The necessary 5 th and 7 th harmonics have a slight deviation of about $0.5 \%$ of the fundamental one compared to simulation results. The current waveform of conventional DQ control should be quite similar to a sinusoidal, as shown in the simulation results in Figure $8 \mathrm{c}$ and harmonic analyses in Figure 8d (left). However, the harmonic spectrum of measured phase currents by the conventional method consist of high 3rd and 4th harmonics due to the high sensitivity of the standard 
method based on the trigonometric transformations to errors in the control system, especially errors in angle measurement.

(a)

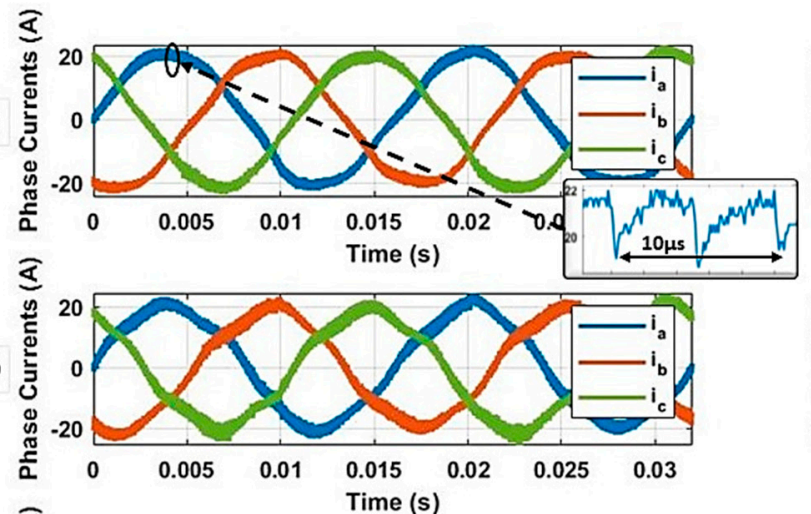

(c)

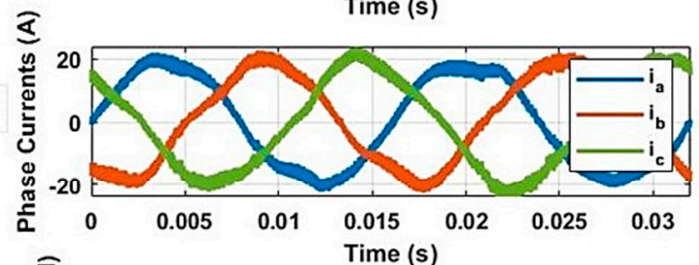

(d)

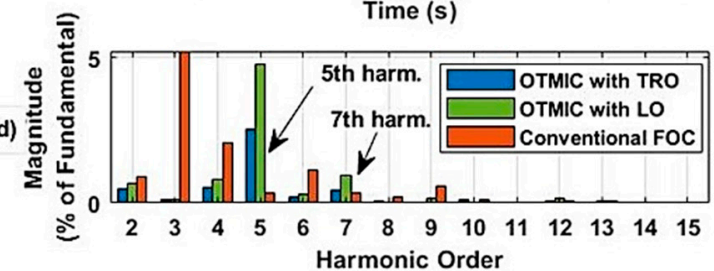

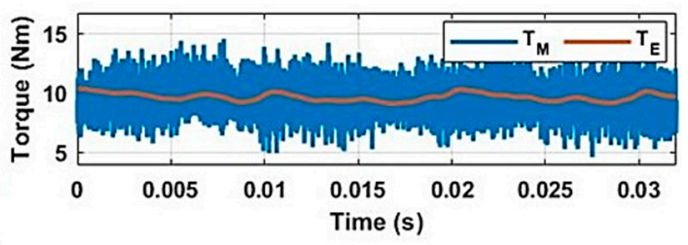
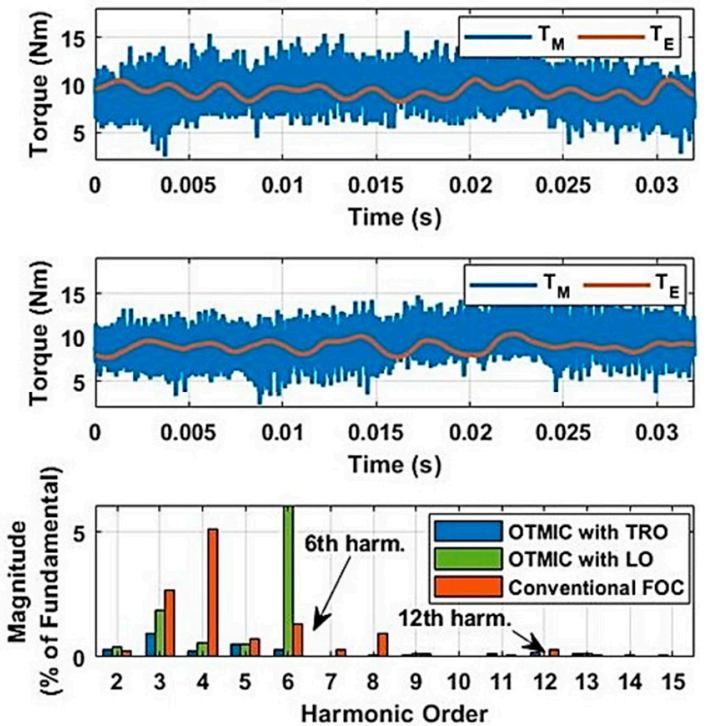

Figure 9. Experimental results of stator current and torque of: (a) OTMIC with torque ripples optimization, (b) OTMIC with losses optimization, (c) conventional DQ control and (d) harmonic spectrum of phase current (left) and torque (right) for every method [18].

The measured torque harmonic distribution shown in Figure $9 \mathrm{~d}$ (right) consists of a large number of harmonics due to speed errors in rotating shaft and additional torque produced by load machine. However, the 6th and 12th harmonics retain their tendency and can be compared with the simulation. In addition, the FFT analyses of measured torques in Figure 9d (right) shows that the proposed OTMIC control with torque ripple minimization is able to compensate additional harmonics in B-field. Summarized, the measured results show very good correspondence to theoretical calculations. As opposed to OTMIC, the Clarke-Park Transformations need a very accurate rotor angle measurement, which can be seen in the larger asymmetries given in Figure 9c. Thus, the proposed OTMIC method is less sensitive to speed fluctuations and angle errors due to the absence of trigonometric transformations of phase currents based on the electric angle.

Figure 10 compares the measured values of motor efficiency for OTMIC control with LO and TRO and conventional DQ control. Motor efficiency was measured with given constant velocity of $30 \mathrm{rad} / \mathrm{s}$, variable torque from 1 to $10 \mathrm{Nm}$ and output power from 30 to $300 \mathrm{~W}$. Results show that OTMIC control with loss minimization increases the system efficiency by $0.7 \%$ in compassion to the conventional method, while delivering the same torque. However, for a correct analysis of the proposed control in case of a motor loss and efficiency, any errors in the measurement system should be avoided and motor torque and current should be increased to the rated values, which could not be fully realized in our experimental setup. More detailed analyses of the system efficiency should be investigated in the future work. 


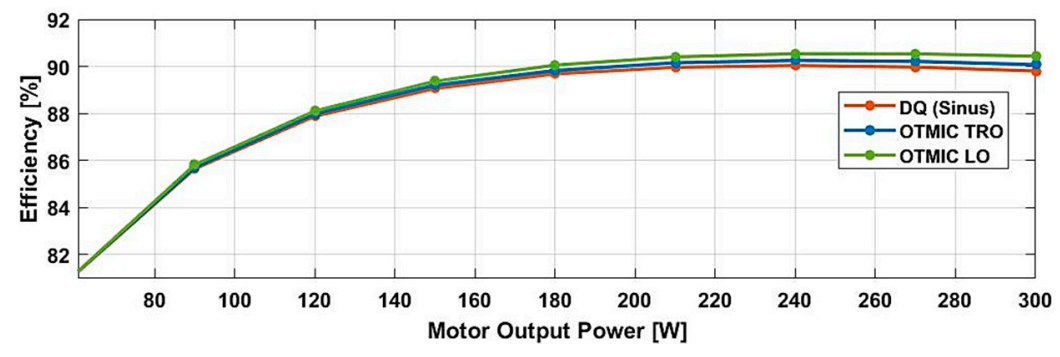

Figure 10. Motor efficiency for OTMIC and DQ control.

Simulation and experimental results show that the OTMIC control system is less sensitive to errors in the electrical angle and phase currents measurements compared to FOC. In addition, the proposed method worked very robustly independent of deviations of motor parameters like phase resistance due to the temperature and the speed of fluctuations due to the load motor.

\section{Conclusions}

This paper proposed a Combined Optimal Torque feedforward and Modal Current feedback Control architecture (OTMIC) for PM motors with very low inductance and higher harmonic B-field, which minimizes motor losses or torque ripples/motor noise by means of offline calculation of optimal current waveform. Optimal three-phase currents are used as reference values to the measured currents. The optimization algorithm considers all relevant coefficients of B-field harmonic distribution to calculate the appropriate current waveform off-line and employs an extremely fast table-lookup for real-time implementation in the microcontroller. Modal current feedback control provides stability, high system dynamics without deviation of a given reference and high robustness against variation of motor parameters or errors in rotor angle measurement.

Compared to classical field-oriented control methods based on the cumbersome and complex Clarke-Park Transformations, the proposed control is simpler for implementation on the microcontroller and allows to minimize either torque ripples or motor losses. As a result, the proposed OTMIC control can reach its full potential in high-frequency systems, where simplicity of the control algorithm and microcontroller calculation time matters. Due to the transformations used in the conventional DQ method, the phase currents' waveforms have to be nearly ideally sinusoidal. This means that conventional methods to achieve the specified requirements of motor torque or total losses demand the special concepts and additional real-time calculations to adapt the phase current waveform to the non-sinusoidal waveform of the magnetic field. It complicates the control system and increases the calculation time in the microcontroller. Moreover, in real high-frequency control systems with errors in current and angle measurements, this problem can be more significant. Experimental results show that the Clarke-Park Transformations for electric drives with a very small motor time constant and high number of poles introduces significant current deviations from the values given. In this case, the proposed OTMIC control method used for PM synchronous motors with a very low phase inductance and tested experimentally shows a very good match between simulated and measured waveforms of phase current and torque. The proposed control can also be applied to other electric motors such as brushless DC (BLDC), asynchronous or PM motors, with any waveform of B-field waveform.

Author Contributions: Conceptualization, R.K. and D.G.; methodology, R.K.; software, R.K. and D.G.; validation, D.G.; formal analysis, R.K. and D.G.; writing-original draft preparation, R.K. and D.G.; writing-review and editing, R.K. and D.G.; funding acquisition, R.K. All authors have read and agreed to the published version of the manuscript.

Funding: This work was supported in part by the European Commission under Grant ERDF (European Regional Development Fund) and the County of Saxony-Anhalt.

Conflicts of Interest: The authors declare no conflict of interest. 


\section{References}

1. Ragot, P.; Markovic, M.; Perriard, Y. Analytical Determination of the Phase Inductances of a Brushless DC Motor with Faulhaber Winding. IEEE Trans. Ind. Appl. 2010, 46, 1360-1366. [CrossRef]

2. Borchardt, N.; Heinemann, W.; Kasper, R. Design of a wheel hub motor with air gap winding and simultaneous utilization of all magnetic poles. In Proceedings of the 2012 IEEE International Electric Vehicle Conference, Greenville, SC, USA, 4-8 March 2012; pp. 1-7.

3. Borchardt, N.; Kasper, R. Nonlinear design optimization of electric machines by using parametric Fourier coefficients of air gap flux density. In Proceedings of the IEEE International Conference on Advanced Intelligent Mechatronics (AIM), Banff, AB, Canada, 12-15 July 2016; pp. 645-650.

4. Kasper, R.; Borchardt, N. Boosting Power Density of Electric Machines by Combining Two Different Winding Types. In Proceedings of the 7th IFAC Symposium on Mechatronic Systems, Leicestershire, UK, 5-8 September 2016; pp. 322-329.

5. Tan, B.; Hua, Z.; Zhang, L.; Fang, C. A New Approach of Minimizing Commutation Torque Ripple for BLDCM. Energies 2017, 10, 173. [CrossRef]

6. Li, X.; Jiang, G.; Chen, W.; Shi, T.; Zhang, G.; Geng, Q. Commutation Torque Ripple Suppression Strategy of Brushless DC Motor Considering Back Electromotive Force Variation. Energies 2019, 12, 1932. [CrossRef]

7. Li, H.; Zheng, S.; Ren, H. Self-Correction of Commutation Point for High-Speed Sensorless BLDC Motor with Low Inductance and Nonideal Back EMF. IEEE Trans. Power Electron. 2017, 32, 642-651. [CrossRef]

8. Li, X.; Xia, C.; Cao, Y.; Chen, W.; Shi, T. Commutation Torque Ripple Reduction Strategy of Z-Source Inverter Fed Brushless DC Motor. IEEE Trans. Power Electron. 2016, 31, 7677-7690. [CrossRef]

9. Yoon, K.-Y.; Baek, S.-W. Robust Design Optimization with Penalty Function for Electric Oil Pumps with BLDC Motors. Energies 2019, 12, 153. [CrossRef]

10. Song, Q.; Li, Y.; Jia, C. A Novel Direct Torque Control Method Based on Asymmetric Boundary Layer Sliding Mode Control for PMSM. Energies 2018, 11, 657. [CrossRef]

11. Qian, J.; Ji, C.; Pan, N.; Wu, J. Improved Sliding Mode Control for Permanent Magnet Synchronous Motor Speed Regulation System. Appl. Sci. 2018, 8, 2491. [CrossRef]

12. Mohd Zaihidee, F.; Mekhilef, S.; Mubin, M. Robust Speed Control of PMSM Using Sliding Mode Control (SMC)-A Review. Energies 2019, 12, 1669. [CrossRef]

13. Wang, Q.; Yu, H.; Wang, M.; Qi, X. A Novel Adaptive Neuro-Control Approach for Permanent Magnet Synchronous Motor Speed Control. Energies 2018, 11, 2355. [CrossRef]

14. Liu, X.; Yu, H.; Yu, J.; Zhao, L. Combined Speed and Current Terminal Sliding Mode Control With Nonlinear Disturbance Observer for PMSM Drive. IEEE Access 2018, 6, 29594-29601. [CrossRef]

15. Zeb, K.; Din, W.U.; Khan, M.A.; Khan, A.; Younas, U.; Busarello, T.D.C.; Kim, H.J. Dynamic Simulations of Adaptive Design Approaches to Control the Speed of an Induction Machine Considering Parameter Uncertainties and External Perturbations. Energies 2018, 11, 2339. [CrossRef]

16. Kang, L.; Cheng, J.; Hu, B.; Luo, X.; Zhang, J. A Simplified Optimal-Switching-Sequence MPC with Finite-Control-Set Moving Horizon Optimization for Grid-Connected Inverter. Electronics 2019, 8, 457. [CrossRef]

17. Kasper, R.; Golovakha, D.; Süberkrüb, F. Combined Optimal Torque and Modal Current Control for Low Inductance PM Motor. In Proceedings of the IEEE International Conference on Mechatronics (ICM), Ilmenau, Germany, 18-20 March 2019; pp. 491-497.

18. Golovakha, D. Combined Optimal Torque and Modal Current Control for Low Inductance PM Motor. Ph.D. Thesis, Otto von Guericke University, Magdeburg, Germany, 2020.

19. Borchardt, N.; Kasper, R. Analytical magnetic circuit design optimization of electrical machines with air gap winding using a Halbach array. In Proceedings of the International Electric Machines and Drives Conference (IEMDC), Miami, FL, USA, 21-24 May 2017; pp. 1-7.

Publisher's Note: MDPI stays neutral with regard to jurisdictional claims in published maps and institutional affiliations. 